Polymer Journal, Vol. 8, No. 6, pp 525-530 (1976)

\title{
Cationic Polymerization of (S)-2-Methylbutyl Propenyl Ether and Optical Rotatory Properties of Its Polymer in Solutino
}

\author{
Yoshitsugu Hirokawa and Toshinobu Higashimura \\ Department of Polymer Chemistry, Faculty of Engineering, \\ Kyoto University, Kyoto 606, Japan.
}

(Received April 23, 1976)

\begin{abstract}
S)-2-Methylbutyl propenyl ether was polymerized into polymers with various stereoregularities by $\mathrm{BF}_{3} \mathrm{O}\left(\mathrm{C}_{2} \mathrm{H}_{5}\right)_{2}$ at low temperature. The relation between the optical activity and the stereoregularity of poly[(S)-2-methylbutyl propenyl ether], which has an asymmetric carbon in $\gamma$-position with respect to the principal chain, was studied in various solvents. The increase of the optical activity of the poly(propenyl ether) with the increase of the stereoregularity was observed in a cyclohexane solution. On the other hand, for poly[(S)-2-methylbutyl vinyl ether], which has no $\beta$-methyl group on the principal chain, the optical activity was independent of the stereoregularity. This difference between the poly(propenyl ether) and the poly(vinyl ether) is undoubtedly related to the presence of a $\beta$-methyl group on the principal chain, which makes the principal chain more rigid. These results suggest the possibility that the stereoregular poly(propenyl ether) takes a specific conformation in solution.

KEY WORDS Propenyl Ether / Poly[(S)-2-methylbutyl propenyl ether] / Poly[(S)-2-methylbutyl vinyl ether] / (S)-2-Methylbutyl Propyl Ether / Cationic Polymerization / Conformation / Optically Active Polymer / Optical Rotatory Dispersion / Stereoregularity /
\end{abstract}

The relation between the optical activity and the stereoregularity of a polymer having an asymmetric carbon atom in the lateral chain has been studied mainly for vinyl polymers ${ }^{1,2}$ such as $\operatorname{poly}\left(\alpha\right.$-olefin), ${ }^{3,4}$ poly(vinyl ketone), ${ }^{5}$ and poly(vinyl ether). ${ }^{6}$ The optical activity of a polymer has been a useful tool to investigate its stereochemistry and conformation. ${ }^{4}$ Pino, et al., ${ }^{3}$ studied optically active poly $(\alpha$-olefin $) s$ and showed that the optical activity of the polymers which had an asymmetric carbon atom in $\alpha-, \beta$-, or $\gamma$-position with respect to the principal chain depended on the stereoregularity of the polymers. This result was explained in terms of the dependence of the polymer conformation on the stereoregularity.

In an optically active poly(vinyl ether) with an asymmetric carbon in $\beta$-position with respect to the principal chain-in other words, in $\alpha$ position with respect to the ethereal oxygen-, the optical activity depended on the stereoregularity of the polymer. However, in contrast to $\operatorname{poly}(\alpha$-olefin), the optical activity of poly(vinyl ether), which has an asymmetric carbon atom in $\gamma$-position with respect to the principal chain, did not depend on the stereoregularity of the polymer. ${ }^{6}$ The difference between the poly(vinyl ether) and the poly( $\alpha$-olefin) has been explained by the suggestion that an ethereal oxygen in the side group of poly(vinyl ether) imparts more flexibility than a methylene group of $\operatorname{poly}(\alpha-$ olefin).

In general, polymers of $\alpha, \beta$-disubstituted olefins must be more rigid than those of monosubstituted ones, because of the steric crowding of the principal chain. Therefore, a stereoregular polymer of an $\alpha, \beta$-disubstituted olefin is expected to form a specific conformation in solution even when a poly ( $\alpha$-substituted olefin) with the same $\alpha$-substituent is in a random 


\section{Y. Hirokawa and T. Higashimura}

conformation. With $\operatorname{poly}(\alpha, \beta$-disubstituted olefin)s, the relation between the optical activity and the stereoregularity has been reported only for poly(2-phenylvinyl alkyl ether) ${ }^{7}$; the optical activity of the polymer was slightly different from that of the model compound and was independent of the distance of the asymmetric carbon atom from the principal chain. However, the relation between the optical activity and the stereoregularity of the polymer was unclear, and the conformation of the polymer was not discussed.

In this paper, the relation between the optical activity and the stereoregularity of the polymer is studied for poly[(S)-2-methylbutyl propenyl ether], which carries both a $\beta$-methyl substituent and an alkoxyl substituent containing an asymmetric carbon atom in $\gamma$-position. This polymer was employed in the investigation because, as with the corresponding poly(vinyl ether), poly[(S)-2methylbutyl vinyl ether], the optical activity of the polymer was found to be almost independent of the stereoregularity of the polymer. ${ }^{6}$

\section{EXPERIMENTAL}

\section{Materials}

(S)-2-Methylbutyl propenyl ether was prepared by transetherification of ethyl propenyl ether and (S)-2-methylbutyl alcohol (optical purity, 92.3\%) in the presence of mercuric acetate. ${ }^{8}$ The geometrical isomers in the product were separated by fractional distillation through a spinning-band column having more than 70 theoretical plates. The boiling point was $127.0^{\circ} \mathrm{C}$ for the cis isomer and $133.0^{\circ} \mathrm{C}$ for the trans isomer. The chemical shift of the $\alpha$-olefinic proton was $5.78 \mathrm{ppm}$ for the cis isomer and $6.13 \mathrm{ppm}$ for the trans isomer, and the spinspin coupling constant of an olefinic $\alpha$ - and $\beta$-proton was $6.5 \mathrm{~Hz}$ for the cis isomer and $13.3 \mathrm{~Hz}$ for the trans isomer, respectively. $[\alpha]_{589}^{20}$ of the monomer was $-0.28^{\circ}$ (neat) for the cis isomer (geometrical purity, 99.0\%) and $+4.28^{\circ}$ (neat) for the trans-rich isomer (cis/trans $=1 / 3$ ).

(S)-2-Methylbutyl vinyl ether was prepared by transetherification of isobutyl vinyl ether and (S)-2-methylbutyl alcohol (optical purity, 92.3\%) in the presence of mercuric acetate..$^{8}$ The boiling point was $112.0^{\circ} \mathrm{C}$ (lit. $\left.{ }^{9} 112^{\circ} \mathrm{C}\right) ;[\alpha]_{589}=+5.40^{\circ}$ (lit. $\left.{ }^{9}[\alpha]_{589}=+5.31^{\circ}\right)$.

The monomers were distilled over $\mathrm{CaH}_{2}$ and then over $\mathrm{Na}$ metal just before use. The purities of these monomers were higher than $99.0 \%$ by gas chromatography.

(S)-2-Methylbutyl propyl ether and (S)-2methylbutyl ethyl ether, model compounds for the optically active polymers, were prepared by hydrogenation on $\operatorname{Pd}(C)$ of the unsaturated precursors. The products were confirmed to be the saturated ethers by NMR and UV spectra. (S)-2-Methylbutyl propyl ether produced from a cis propenyl ether exhibited the same optical activity as that from a trans-rich isomer.

$\mathrm{BF}_{3} \mathrm{O}\left(\mathrm{C}_{2} \mathrm{H}_{5}\right)_{2}$ and solvents were purified by the conventional methods.

\section{Procedures}

The polymerization was carried out in a flask equipped with a three-way stopcock under dry nitrogen. The reaction was stopped by addition of methanol. Conversion was determined by measuring the concentration of residual monomers by gas chromatography and by weighing the methanol-insoluble polymers. The polymers were reprecipitated several times with methanol from toluene.

Intrinsic viscosity of the polymer was measured in benzene solution at $30^{\circ} \mathrm{C}$. ORD was measured in polymer solutions (concn, 2.0$3.0 \mathrm{~g} / \mathrm{d} l)$ at $20^{\circ} \mathrm{C}$ with a JASCO J-20-type polarimeter (cell length, $0.2 \mathrm{dm}$ ).

\section{RESULTS AND DISCUSSION}

\section{Polymerization of (S)-2-Methylbutyl Propenyl Ether}

Table I shows the polymerization conditions of (S)-2-methylbutyl propenyl ether and the characteristics of the polymers obtained. (S)2-Methylbutyl propenyl ether was easily polymerized, giving a white powdery polymer in a high yield at low temperatures. Obviously neither a $\beta$-methyl group nor a bulky alkoxyl group interfered with the polymerization. (S)2-Methylbutyl propenyl ether produced polymers of higher molecular weight $([\eta]=0.30-0.45)$ than poly(1-methylpropyl propenyl ether) $([\eta]=$ ca. 0.1) under the same conditions. This appears to be related to the fact that the side chain of the former branches far from the double bond and exerts a smaller steric hindrance against 
Optically Active Poly(2-Methylbutyl Propenyl Ether)

Table I. Polymerization of (S)-2-methylbutyl propenyl ether ${ }^{a}$

\begin{tabular}{cclcccc}
\hline Run & $\begin{array}{c}\text { Monomer, } \\
\text { cis/trans }\end{array}$ & Solvent & $\begin{array}{c}\text { Yield, } \\
\%\end{array}$ & $\begin{array}{c}{[\eta],} \\
\mathrm{d} l / \mathrm{g}\end{array}$ & $D_{1150} / D_{1400}$ & Crystallinity $^{\mathrm{b}}$ \\
\hline 1 & $25 / 75$ & $\mathrm{C}_{6} \mathrm{H}_{5} \mathrm{CH}_{3}$ & 83 & 0.31 & 3.98 & ++ \\
2 & $25 / 75$ & $\mathrm{CH}_{2} \mathrm{Cl}_{2}$ & 99 & 0.35 & 3.23 & + \\
3 & $100 / 0$ & $\mathrm{C}_{6} \mathrm{H}_{5} \mathrm{CH}_{3}$ & 97 & 0.44 & 2.95 & - \\
4 & $100 / 0$ & $\mathrm{CH}_{2} \mathrm{Cl}_{2}$ & 96 & 0.37 & 3.12 & - \\
\hline
\end{tabular}

a Temp, $-78^{\circ} \mathrm{C}$; catalyst, $\mathrm{BF}_{3} \mathrm{OEt}_{2} ;[\mathrm{M}]_{0}, 10 \mathrm{vol} \%$; $[\mathrm{C}]_{0}, 2.0 \mathrm{mM}$; time, $22 \mathrm{hr}$.

b X-ray diffraction.

the polymerization than the latter.

The stereoregularity of the polymer was determined by use of X-ray diffraction and IR spectra. This polymer has so many protons that the stereoregularity of the polymers could not be quantitatively determined by NMR spectra. The ratio of the optical density of $1150 \mathrm{~cm}^{-1}$ against $1400 \mathrm{~cm}^{-1}$ was calculated as a measure of the stereoregularity of the polymer, because the optical density ratio was in good correspondence with the degree of crystallinity observed by X-ray diffraction.

As Table I shows, when a trans-rich monomer was polymerized in toluene, a highly stereo- regular polymer was produced. On the other hand, a cis monomer produced only an amorphous polymer. This tendency has been often observed with other alkyl propenyl ethers. ${ }^{10}$ Therefore, it can be considered that the steric structure of the crystalline polymer obtained from a trans-rich monomer may be the threodiisotactic structure. ${ }^{10}$

\section{ORD of Poly[(S)-2-Methylbutyl Propenyl Ether]}

ORD of poly[(S)-2-methylbutyl propenyl ether]s with various stereoregularities was measured in cyclohexane. Figure 1 shows the ORD curves of poly[(S)-2-methylbutyl propenyl ether] and

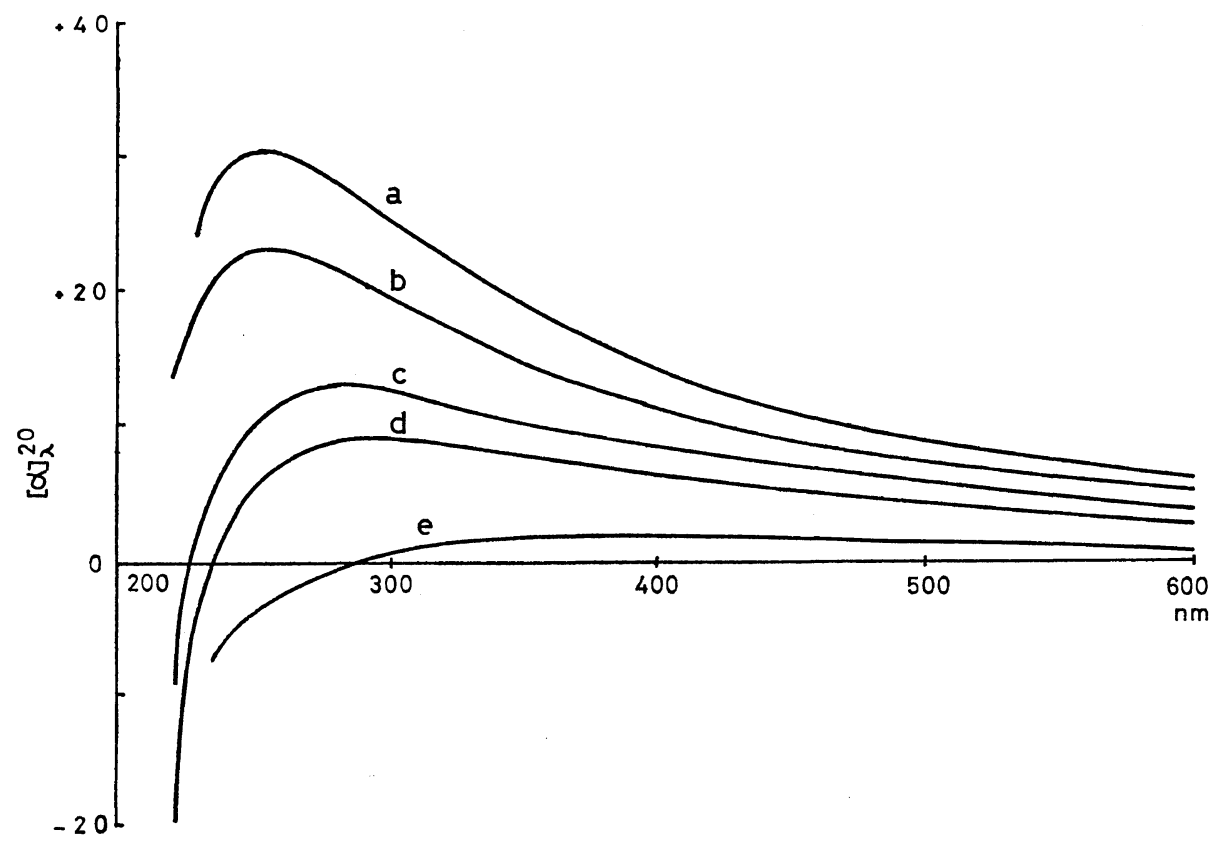

Figure 1. ORD curves of poly[(S)-2-methylbutyl propenyl ether] in cyclohexane. Starting monomer and polymerization solvent: (a), trans-rich, toluene; (b), trans-rich, methylene dichloride; (c), cis, methylene dichloride; (d), cis, toluene; (e), model compound, (S)-2-methylbutyl propyl ether. 


\section{Y. Hirokawa and T. Higashimura}

the corresponding model compound, (S)-2- dicates that the optical activity of the polymer methylbutyl propyl ether, in cyclohexane. All polymers showed a larger optical activity than the model compound. The maxima in the ORD curves were observed in the range from $250 \mathrm{~nm}$ to $300 \mathrm{~nm}$. The same ORD curve pattern has been observed with the corresponding poly(vinyl ether)s. ${ }^{2}$ The one-term Drude equation did not hold for the ORD curves. Figure 1 also independs upon the degree of stereoregularity.

In order to investigate whether the optical activity of the polymer is conformation-dependent, the ORD was measured in solvents other than cyclohexane. In contrast to the result in cyclohexane solution, the optical activities of the polymer in toluene and methylene dichloride were almost independent of the

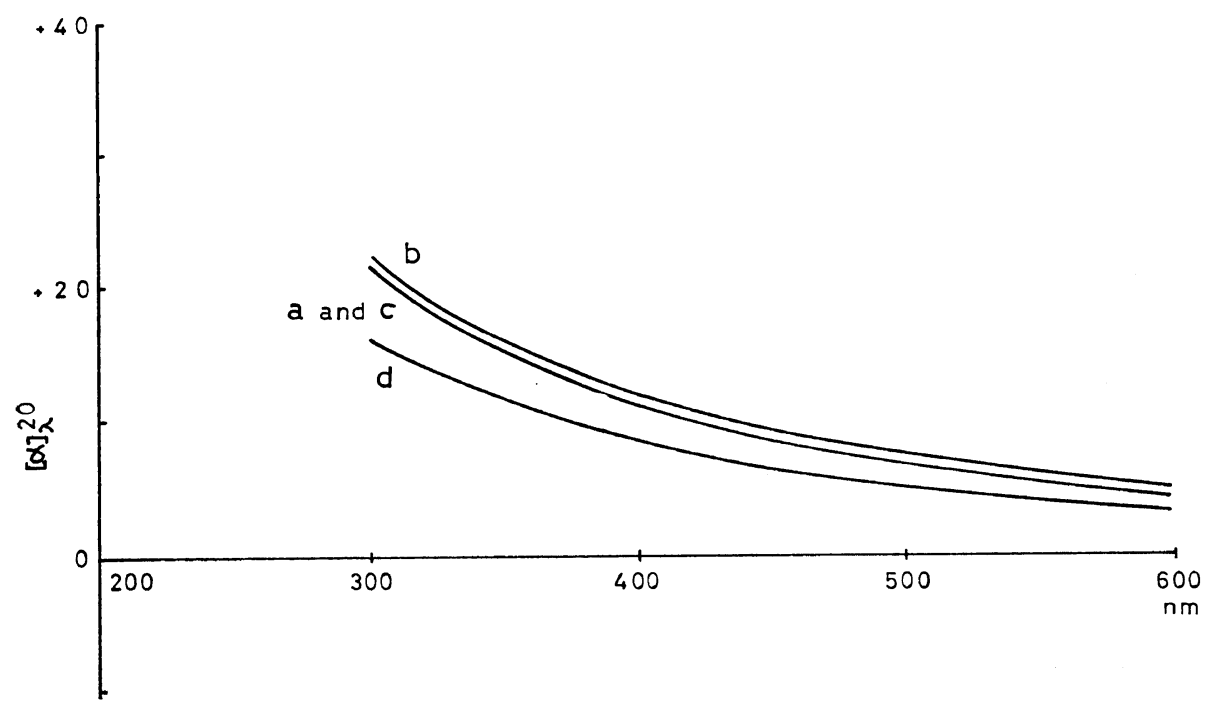

Figure 2. ORD curves of poly[(S)-2-methylbutyl propenyl ether] in toluene. Designations for starting monomers and polymerization solvents are the same as in Figure 1.

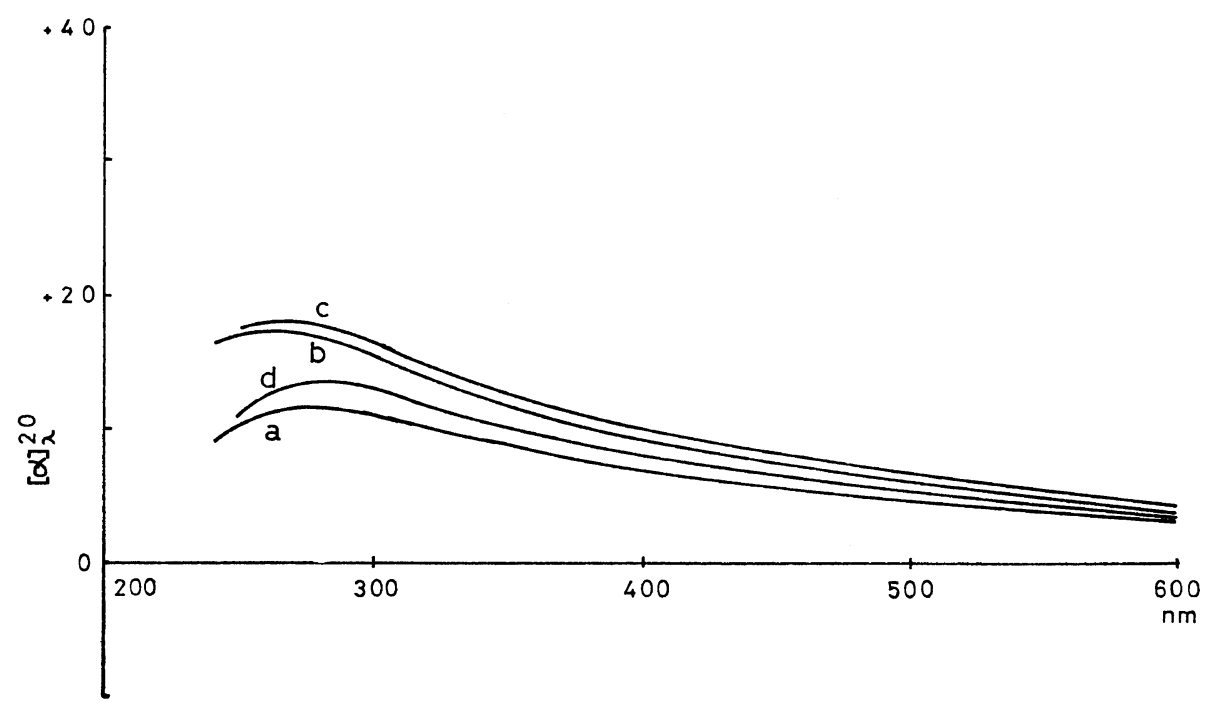

Figure 3. ORD curves of poly[(S)-2-methylbutyl propenyl ether] in methylene dichloride. Designations for starting monomers and polymerization solvents are the same as in Figure 1. 


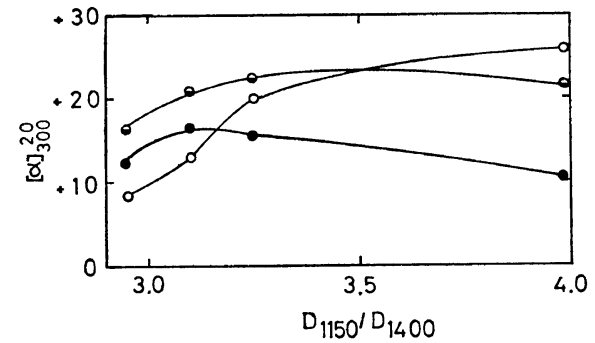

Figure 4. Plot of $[\alpha]_{300}^{20} v s . D_{1150} / D_{1400}$ of poly[(S)-2methylbutyl propenyl ether]: $(\bigcirc)$, in cyclohexane; $(\ominus)$, in toluene; $(\odot)$, in methylene dichloride.

geometrical structure of the starting monomer and the kind of the solvent used for the polymerization, as shown in Figures 2 and 3.

Figure 4 shows the plot of $[\alpha]_{300}^{20}$ against the index for stereoregularity, which was obtained from IR spectra of the polymer. The optical activity was nearly independent of the stereoregularity of the polymer in toluene or methylene dichloride, but in cyclohexane the optical activity increased as the stereoregularity increased. These results suggest that, owing to a $\beta$-methyl group and a bulky alkoxyl group, the principal chain of poly[(S)-2-methylbutyl propenyl ether] is so rigid that a stereoregular polymer takes a specific conformation even in a weakly solvating medium such as cyclohexane. A similar observation has been made with poly(tert-butyl crotonate) obtained by anionic polymerization. ${ }^{11}$ The specific conformation of poly[(S)-2-methylbutyl propenyl ether] is definitely induced by the rigidity of the principal chain and the chirality in the lateral chain. On the other hand, in strongly solvating media such as toluene and methylene dichloride, poly[(S)- 2-methylbutyl propenyl ether] does not seem to assume any specific conformation.

\section{Comparison between Poly[(S)-2-methylbutyl pro- penyl ether $]$ and Poly[(S)-2-methylbutyl vinyl ether]}

It is thought that a stereoregular poly[(S)-2methylbutyl propenyl ether) formed a specific conformation induced by the chirality of the lateral chain in cyclohexane. But, Pino, et al., ${ }^{6}$ have reported that poly[(S)-2-methylbutyl vinyl ether]s with various stereoregularities exhibited only slight differences in their optical activities in isooctane in a short wavelength region. Table II summarizes the polymerization conditions and the characteristics of poly[(S)-2-methylbutyl vinyl ether]. The polymerization of (S)-2methylbutyl vinyl ether was faster than the corresponding propenyl ether and the molecular weight of the polymer was much larger than the corresponding poly(propenyl ether). The stereoregularity of the poly(vinyl ether) was determined by X-ray diffraction and IR spectra. The ratio of the optical densities at $1100 \mathrm{~cm}^{-1}$ against $1080 \mathrm{~cm}^{-1}$ was used as a measure of the stereoregularity of the polymer. The optical density ratio was in good correspondence with the degree of crystallinity determined by X-ray diffraction.

Figure 5 shows the ORD curves of $\operatorname{poly}[(\mathbf{S})$ 2-methylbutyl vinyl ether]s with various stereoregularities and of the model compound, (S)2-methylbutyl ethyl ether. The optical activity of the polymer was larger than that of the model compound. But the differences of the optical activities among the polymers with different stereoregularities were not very significant. These results coincide with the previous report ${ }^{6}$

Table II. Polymerization of (S)-2-methylbutyl vinyl ether ${ }^{a}$

\begin{tabular}{clccccc}
\hline Run & Solvent & $\begin{array}{c}\text { Temp, } \\
{ }^{\circ} \mathrm{C}\end{array}$ & $\begin{array}{c}\text { Yield, } \\
\%\end{array}$ & $\begin{array}{c}{[\mathrm{g}],} \\
\mathrm{d} l / \mathrm{g}\end{array}$ & $D_{1100} / D_{1080}$ & Crystallinity $^{\mathrm{b}}$ \\
\hline $5^{\mathrm{c}}$ & $n-\mathrm{C}_{6} \mathrm{H}_{14}$ & -78 & 96 & 1.74 & 1.10 & ++ \\
6 & $\mathrm{C}_{6} \mathrm{H}_{5} \mathrm{CH}_{3}$ & -78 & 99 & 1.36 & 1.02 & + \\
7 & $\mathrm{CH}_{2} \mathrm{Cl}_{2}$ & -78 & 96 & 1.30 & 0.95 & - \\
8 & $\mathrm{C}_{6} \mathrm{H}_{5} \mathrm{CH}_{3}$ & -20 & 95 & 1.01 & 0.96 & - \\
9 & $\mathrm{CH}_{2} \mathrm{Cl}_{2}$ & -20 & 90 & 0.71 & 0.87 & - \\
\hline
\end{tabular}

a Catalyst, $\mathrm{BF}_{3} \mathrm{OEt}_{2} ;[\mathrm{M}]_{0}, 10$ vol \%; $[\mathrm{C}]_{0}, 2.0 \mathrm{mM}$; Time, $6 \mathrm{hr}$.

b X-ray diffraction.

c $[\mathrm{M}]_{0}, 4.0$ vol \%; $[\mathrm{C}]_{0}, 5.0 \mathrm{mM}$. 


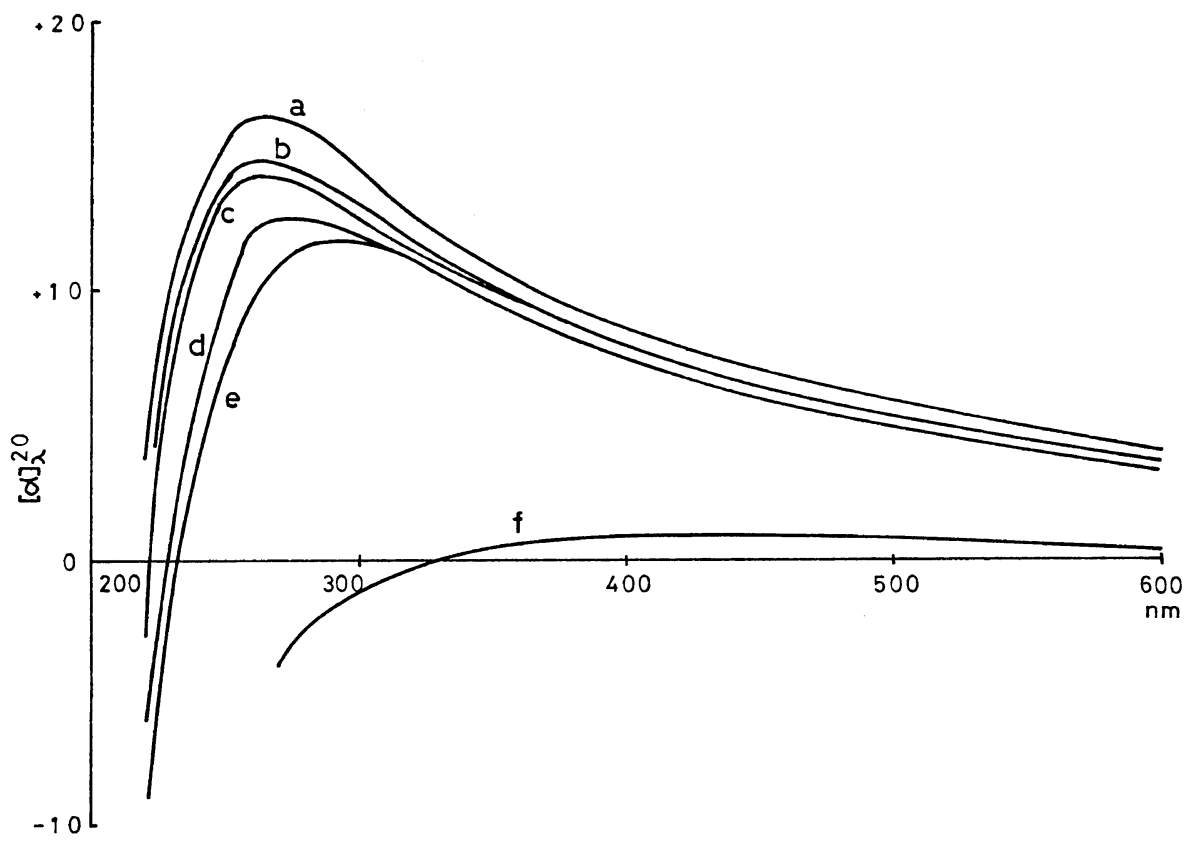

Figure 5. ORD curves of poly[(S)-2-methylbutyl vinyl ether] in cyclohexane. Polymerization temperature and solvent: (a), $-78^{\circ} \mathrm{C}, n$-hexane; (b), $-78^{\circ} \mathrm{C}$, toluene; (c), $-78^{\circ} \mathrm{C}$, methylene dichloride; (d), $-20^{\circ} \mathrm{C}$, toluene; (e), $-20^{\circ} \mathrm{C}$, methylene dichloride; (f), model compound, (S)-2-methylbutyl ethyl ether.

that with poly[(S)-2-methylbutyl vinyl ether] the stereoregularity has little effect on the conformation in aliphatic solvents.

To conclude, the optical activity of poly[(S)2-methylbutyl propenyl ether], which has an asymmetric carbon in $\gamma$-position with respect to the principal chain, was found to increase with increase of the stereoregularity. This observation is in contrast with that for poly[(S)2-methylbutyl vinyl ether]. The difference between the poly(propenyl ether) and the poly(vinyl ether) must be due to the presence of a $\beta$-methyl group on the principal chain, which plays an important role in limiting the conformation of poly(propenyl ether) in solution.

\section{REFERENCES}

1. For a review, see P. Pino, Advan. Polym. Sci., 4, 393 (1965).

2. For a review, see P. Pino, P. Salvadori, E. Chiellini, and P. L. Luisi, Pure Appl. Chem.,
16, 469 (1968).

3. See for instance, P. Pino, F. Ciardelli, G. P. Lorenzi, and G. Montagnoli, Makromol. Chem., 61, 207 (1963).

4. P. Pino and P. Neuenschwander, J. Polym. Sci., Polym. Symp. Ed., No. 51, 171 (1975).

5. O. Pieroni, F. Ciardelli, C. Botteche, L. Lardicci, P. Salvadori, and P. Pino, ibid., Part C, No. 22, 993 (1969).

6. P. Pino, G. P. Lorenzi, and E. Chiellini, ibid., Part C, No. 16, 3279 (1968).

7. R. Vukorić and D. Fleś, ibid., Polym. Chem. Ed., 13, 49 (1975).

8. W. H. Watanabe and L. E. Conlon, J. Amer. Chem. Soc., 79, 2828 (1957).

9. E. Chiellini and M. Marchetti, Makromol. Chem., 169, 59 (1973).

10. G. Natta, J. Polym. Sci., 48, 219 (1960); T. Higashimura, S. Kusudo, Y. Ohsumi, A. Mizote, and S. Okamura, J. Polym. Sci., Part A-1, 6, 2511 (1968).

11. T. Kitano, T. Fujimoto, and M. Nagasawa, Macromolecules, 7, 719 (1974). 